\title{
The dual challenge of BP control
}

$\mathrm{H}$ ypertension is a huge global health concern. In 2002, the WHO reported that this condition was the cause of 7.1 million deaths ( $13 \%$ of the total) and represented $4.5 \%$ of the disease burden worldwide. Crucially, elevated blood pressure was the risk factor associated with the highest mortality in both low-income and high-income countries (WHO. World Health Report. Geneva; 2002). The global burden of hypertension must be reduced to prevent an impending long-term public health problem. Evidence has now emerged that the burden of hypertension may have been underestimated, at least in developing nations, and that, once identified, the condition is seriously undertreated. We are, therefore, confronted with a dual challenge of identifying and appropriately treating hypertension.

High blood pressure has long been recognized as one of the primary risk factors for cardiovascular disease, and is particularly associated with stroke and myocardial ischemia. In 2000, an estimated one in four adults worldwide (972 million individuals, or $26.4 \%$ of the population) had 'hypertension', defined as blood pressure above $140 / 90 \mathrm{mmHg}$ (Kearney, P. M. et al. Lancet $365,217-223$; 2005). However, cardiovascular risk begins to increase incrementally at pressures as low as $115 / 75 \mathrm{mmHg}$ and mortality from ischemic heart disease and stroke doubles for every $20 \mathrm{mmHg}$ increase in systolic blood pressure and $10 \mathrm{mmHg}$ increase in diastolic blood pressure in individuals aged 40-89 years. The seventh report of the Joint National Committee on Prevention, Detection, Evaluation, and Treatment of Hypertension (JNC7) classified 'normal' blood pressure as being below $120 / 80 \mathrm{mmHg}$, and pressures in the range 120-139/ 80-89 mmHg as 'prehypertension'. Clearly, applying these classifications substantially increases the estimated number of individuals worldwide whose blood pressure puts them at risk of cardiovascular disease. Moreover, in the next 15 years, total population growth and ageing of the population are expected to result in a huge rise in the number of individuals with hypertension-to around 1.5 billion worldwide. In low-income countries alone, the number of people with hypertension is predicted to increase by 500 million by 2025 (Perkovic, V. et al. Hypertension 50, 991-997; 2007).

A report from the China National Hypertension Survey Epidemiology Follow-Up Study demonstrates the scale of the hypertension problem and makes uneasy reading (He, J. et al. Lancet doi:10.1016/S01406736(09)61489-6). The investigators found that around 2.11 million cardiovascular deaths among Chinese adults in 2005 were attributable to raised blood pressure.
This figure is substantially higher than the 1.8 million deaths that could have been predicted on the basis of previous analyses, and could still be an underestimate of true hypertension-related mortality because the prevalence of uncontrolled blood pressure is higher now than in the 1990s when the survey was conducted. Another worrying aspect of this finding is that, on the basis of previous surveys, only $24 \%$ of Chinese individuals with hypertension are aware of their condition and only $19 \%$ receive evidence-based therapy.

The implementation of effective management strategies is the second vital step toward reducing the incidence of hypertension. Treatment should focus on achieving a target blood pressure, within the levels suggested by the JNC7 report, and according to published guidelines. The most effective way of reducing blood pressure is to implement simple lifestyle changes, such as eating a healthy diet (including reducing alcohol and salt intake) and taking regular exercise. Such interventions can reduce the need for and increase the benefits of antihypertensive drug therapy and dramatically reduce cardiovascular risk. However, hypertension control remains suboptimal in most areas.

This reality was highlighted by Pereira and colleagues in their systematic review of 44 studies from 35 countries. In developing nations, although the prevalence of hypertension was reported to be $32.2 \%$ for men and $30.5 \%$ for women, the control rates were just $9.8 \%$ and $16.2 \%$ of those with hypertension for men and women, respectively. The figures were very similar for developed countries where the prevalence was $40.8 \%$ for men and $33.0 \%$ for women, with control rates for those with hypertension of just $10.8 \%$ and $17.3 \%$ (Pereira, M. et al. J. Hypertens. $27,963-975 ; 2009)$. Low-income countries face additional barriers such as lack of national treatment guidelines, low availability of trained medical personnel, lack of educational programs, and political issues related to health care. Although incremental decreases in systolic blood pressure are correlated with reductions in the incidence of cardiovascular events, antihypertensive treatment does not completely eliminate cardiovascular risk. Therefore, primary prevention-in the form of lifestyle interventions such as weight loss, reduced dietary salt and alcohol intake-is also critical.

Researchers from Duke University, USA have demonstrated the effectiveness of a simple, cost-effective, patient-managed hypertension management strategy (Bosworth, H. B. et al. Ann. Intern. Med. 151 (10), advance online publication 5 October 2009. Home blood pressure monitoring combined with a nurse-led behavioral

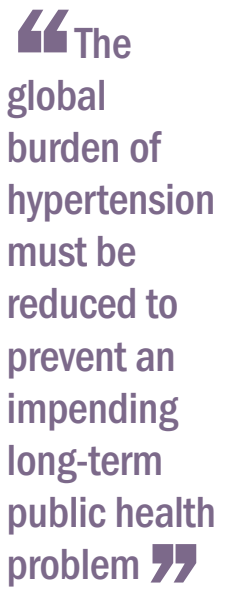

Valentin Fuster is the Editor-in-Chief and Alexandra King is Senior Editor of Nature Reviews Cardiology.

Competing interests The authors declare no competing interests. 
intervention, which involved regular moderate-intensity physical activity, smoking cessation, weight loss, and reduced alcohol and salt intake, was associated with an $11 \%$ improvement in the number of patients with reasonably controlled blood pressure $(140 / 90 \mathrm{mmHg})$ after 2 years when compared with standard care. In addition, patients in the combined intervention group had a mean blood pressure reduction of $3.9 \mathrm{mmHg}$, whereas no reduction was observed the standard-care group. This study highlights the relevance of empowering patients to make lifestyle modifications as part of a comprehensive blood pressure control initiative.

Driving down the prevalence of high blood pressure is a worldwide public health priority, now and for the future. Only by addressing the double challenge of identification and effective management of hypertension can we reduce the cardiovascular mortality attributable to this eminently preventable risk factor.

doi:10.1038/nrcardio.2009.202 